\title{
SUR LES TRÉMATODES D'EMYS ORBICULARIS L.
}

\author{
Par le professeur K,-I. SKRIABINE
}

De la Section helminthologique de I'Institut expérimental vétérinaire à Moscou

Ayant organisé, en automne 1923, une expédition helminthologique pour l'étude de la faune des vers parasites de l'Arménie, j'avais marqué dans mon programme, entre autres iquestions, l'étude des parasites des reptiles de la Transcaucasie. A la fin du mois d'août, pendant les travaux de l'expédition dans la vallée de la rivière Araxe, nous avons disséqué, à la station Schachtachti, 6 exemplaires d'Emys orbicularis L. Toutes ces tortues, sans exception, étaient envahies par des vers parasites et trois d'entre elles par des trématodes. Ayant examiné ces trématodes, nous les avons rapportés à trois espèces: Telorchis solivagus Odhner, 1902, Patagium lazarevi Skriabine et Popov, 1925, et Polystomum ocellatum (Rud.).

L'intensité de l'infection par ces trématodes était la suivante : une tortue renfermait 286 exemplaires des trois espèces, la seconde renfermait deux espèces: Patagium et Telorchis et enfin, chez la troisième tortue, il fut constaté seulement une espèce : Telorchis solivagus:

Je dois ajouter que les dissections furent opérées conformément à la méthode spéciale helminthologique, laquelle permet de constater la présence d'absolument tous les parasites chez l'hôte étudié.

\section{Polystomum ocellatum (Rud.)}

J'ai trouvé un exemplaire de ce parasite dans la vessie urinaire d'Emys orbicularis L. et, cependant, il est normal que cette espèce se localise, chez les tortues, dans le pharynx. Pour éclaircir cette nouvelle localisation du parasite, je dois faire l'hypothèce suivante : On sait que $P$. integerrimum, l'espèce voisine de $P$. ocellatum, habite à son stade larvaire sur les branchies des têtards. Après la transformation du têtard en grenouille, la larve de ce trématode passe dans le tube digestif de son hôte, traverse l'intestin et pénètre dans la vessie urinaire, où elle devient adulte.

2. Patagium lazarevi Skriabine et Popov, 1925

HôtE. - Emys orbicularis L.

Localisation. - Duodénum.

Annales de Parasitologie, $\mathrm{T}$, III, $\mathrm{x}^{\circ} 3$. - Juillet 1925, p. 281-289.

19. 
Distribution gÉographigue. - Arménie, vallée de la rivière Araxe, station Schachtachti.

InTENSITÉ DE L'INFECTION. - Sur 6 Emys disséqués, cette espèce

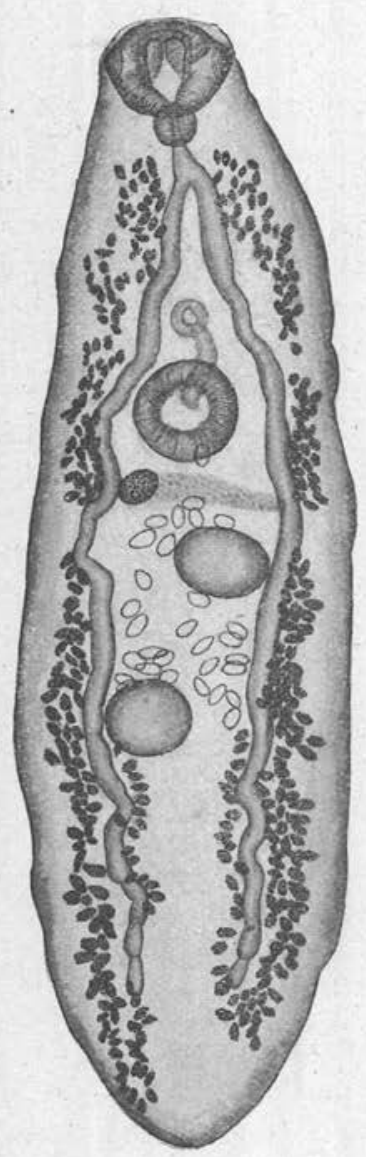

Fig. 1. - Palagium lazarevi Skriabine et Popov, 1925. fut constatée deux fois, ce qui fait un pourcentage de 33,3 d'individus infestés.

DEscription. - Le corps du parasite est oblong et ovale; l'extrémité antérieure et l'extrémité postérieure sont plus étroites. La longueur du corps est de $2 \mathrm{~mm}$., 6, la largeur maximum, dans la région des testicules, est de $0 \mathrm{~mm}$., 79 . La ventouse buccale est caractérisée, dans le genre Patagium, par sa forme hexagonale à angles arrondis ; deux formations triangulaires coniques de forme auriculaire passent à droite et à gauche de son bord antérieur. L'entrée de la ventouse est triangulaire. La longueur de la ventouse buccale est de $0 \mathrm{~mm}$., 21 , la largeur de $0 \mathrm{~mm}$., 29. Le pharynx est rond et de $0 \mathrm{~mm}$., 09 de diamètre. L'œsophage est court. Les branches de l'intestin dépassent la limite postérieure des testicules, n'atteignant pas cependant l'extrémité postérieure du corps.

La ventouse ventrale a $0 \mathrm{~mm}$., 25 de diamètre ; son centre se trouve à la distance de $0 \mathrm{~mm}$., 93 de l'extrémité antérieure du corps. Les testicules sont situés dans la partie centrale du parasite, obliquement l'un par rapport à l'autre, le testicule antérieur étant tout près du bord interne de la branche gauche de l'intestin et le testicule postérieur tout près de la branche droite. Le testicule antérieur se trouve juste au milieu de la longueur du corps, le testicule postérieur est séparé du testicule antérieur par un intervalle considéráble occupé par les anses de l'utérus. Le testicule antérieur mesure $0 \mathrm{~mm}$., $17 \times 0 \mathrm{~mm}$., 2 et le postérieur $0 \mathrm{~mm}$., $19 \times 0 \mathrm{~mm}$., 19 .

L'ovaire, plus petit que les testicules, est situé près du bord interne de la branche droite de l'intestin, en avant du testicule antérieur, en arrière de la ventouse ventrale, il mesure $0 \mathrm{~mm}$., 09 sur $0 \mathrm{~mm}$., 1. La 
poche du cirrhe atteint par son fond le niveau du milieu de la ventouse ventrale, en y comprenant la vésicule séminale, clairement visible. L'orifice génital est enveloppé d'un manchon musculeux. L'utérus est court et développe ses circonvolutions principalement entre la ventouse ventrale et le testicule postérieur ; il ne s'étend pas jusqu'à la limite postérieure de ce dernier.

Les vitellogènes, assez grands, commencent immédiatement après le pharynx et s'étendent jusqu'à l'extrémité postérieure du corps, dépassant les extrémités cæcales de l'intestin. A la hauteur de l'ovaire, de chaque côté, il y a interruption des vitellogènes, ce qui forme de chaque côté un groupe de vitellogènes antérieur et un groupe postérieur.

Les glandes vitellogènes postérieures commençant au niveau du testicule antérieur, s'étendent latéralement à l'intestin ; ayant atteint l'extrémité cæcale de l'intestin; elles la contournent sur le côté interne en remontant antérieurement jusqu'au niveau du testicule postérieur.

En somme, dans la partie postérieure du corps du parasite, nous avons comme 4 lignes de glandes vitellogènes : 2 en dehors et 2 en dedans des branches de l'intestin. Les deux rangées internes de vitellogènes sont séparées par le trone postérieur de l'appareil excréteur.

Les œufs, de couleur jaune pâle, mesurent $0 \mathrm{~mm}$., 08 de longueur et $0 \mathrm{~mm}$., 04 de largeur.

Position systématique du parasite. - Jusqu’à présent il n'a été décrit qu'une espèce du genre Patagium : P. brachydelphium Heymann, 1905, de Dermatemys mawi Gray, avec laquelle notre espèce a une assez grande ressemblance; elle en diffère cependant par les caractères suivants :

$1^{\circ}$ Les testicules de notre espèse sont écartés très nettement, tandis que chez $P$. brachydelphium ils se touchent presque l'un l'autre.

$2^{\circ}$ L'ovaire de notre espèce est plus de deux fois plus petit que celui de $P$. brachydelphium.

$3^{\circ}$ La poche du cirrhe de notre espèce est moins développée que chez $P$. brachydelphium.

$4^{\circ}$ Le testicule antérieur de notre espèce est situé tout entier dans la partie antérieure du corps (la limite passe juste au niveau de son bord postérieur), tandis que le testicule antérieur de $P$. brachydelphium se trouve entièrement dans la moitié postérieure du corps.

$5^{\circ}$ Les glandes vitellogènes de notre espèce sont moins développées que chez $P$. brachydelphium. 


\begin{tabular}{|c|c|c|}
\hline & $\begin{array}{c}\text { Patagiem Brachydelphivm } \\
\text { Hgyman, } 1905\end{array}$ & $\begin{array}{l}\text { Patagivm lazarevi } \\
\text { Skmiabine et Popov, } 1925\end{array}$ \\
\hline Long. du corps.. & 2,73 & 2,6 \\
\hline Largeur maxi- & & \\
\hline ma du corps... & 0,6 & 0,79 \\
\hline Ventouse buccale & 0,246 & $0,21 \times 0,29$ \\
\hline Ventouseventrale & & \\
\hline diamètre...... & 0,252 & 0,25 \\
\hline Pharynx ......... & $0,114 \times 0,072$ & 0,09 \\
\hline $\begin{array}{r}\text { Diamètre des tes- } \\
\text { ticules.......... }\end{array}$ & 0,252 et 0,210 & $0,17 \times 0,2 ; 0,19 \times 0,19$ \\
\hline $\begin{array}{l}\text { Ovaire, la rge ur } \\
\text { maxima........ }\end{array}$ & 0,210 & $0,09 \times 0,1$ \\
\hline Gufs............ & $0,078 \times 0,042$ & $0,08 \times 0,04$ \\
\hline Hôte ............ & Dermalemys mavi Gray & Emys orbicularis L. \\
\hline graphique...... & $?(1)$ & Arménie, Vallée de la rivière Araxe \\
\hline
\end{tabular}

Ce parasite a été nommé Patagium lazarevi en l'honneur du $D^{r}$ S. M. Lazarev, le commissaire de la défense de la santé du peuple en Arménie, qui a rendu de grands services à la dixième expédition helminthologique pendant ses travaux.

\section{Telorchis solivagus Odhner, 1902}

En 1902, Odhner, dans son travail " Trematoden aus Reptilien nebst allgemeinen systematischen Bemerkungen ", publié dans "Ofversigt af Kongl. Vetenskaps-Akademiens Fbrhandlingar, 59, $\mathrm{N}^{\circ}$ 1, 1902 ", a décrit Telorchis solivagus n. sp., de Transcaucasie ; un exemplaire unique de cette espèce fut trouvé dans le duodénum d'une tortue Clemmys caspica récoltée par le $\mathrm{D}^{\mathrm{r}}$ Lönnberg pendant son voyage en Transcaucasie (la localité exacte n'est pas indiquée) et envoyée vivante en Suède (Upsala). Depuis lors, personne n'a retrouvé ce parasite.

(1) G. Heymann n'a pas indiqué la provenance de l'hôte de son Patagium, mais rappelons que Dermatemys mawi Gray, 1847 qui appartient, parmi les chéloniens thécophores, à la superfamille des Cryptodira, famille des Dermatemydæ Gray est une espèce de l'Amérique Centrale (Tabasco, Guatemala, Yucatan, Bélize, Honduras, d'après le catalogue de Boulenger). La comparaison de Patagium brachydelphinm Heymann, 1905 avec $P$. lazarevi Skriabine, 1925 ne permet de déceler que des différences extrêmement faibles et il est très intéressant de constater que le genre Palagium, que l'on pouvait jusqu'à présent considérer comme américain, est représenté, en Asie Occidentale, par une espèce extrêmement voisine de celle d'Amérique.

[Robert-Ph. Dollfus . 
Mon expédition de 1923 a recueilli ce parasite dans le duodénum d'un hôte nouveau : Emys orbicularis L. en Arménie, au bord de la rivière Araxe (station Schachtachti).

INTENSITÉ DE L'INFECTION. - Sur 6 Emys orbicularis disséqués, le parasite fut observé trois fois, ce qui fait une proportion de $500 / 0$ d'individus infestés.

Description. - Le corps, excessivement allongé, atteint $9 \mathrm{~mm}$., 5 de longueur. La largeur du corps est la même sur la plus grande partie de sa longueur, s'élargissant un peu au niveau de la ventouse ventrale et se rétrécissant vers l'extrémité antérieure. La largeur maxima, dans la région de la ventouse ventrale, est de $0 \mathrm{~mm} ., 8$.

Dans sa partie antérieure, le corps est couvert par de petits écussons cuticulaires rapprochés. La ventouse buccale a $0 \mathrm{~mm}$., 158 de diamètre. Le pharynx atteint $0 \mathrm{~mm}$., 099 de long. Les branches de l'intestin s'étendent jusqu'à l'extrémité du corps, dépassant la limite du testicule postérieur. La ventouse ventrale a $0 \mathrm{~mm}$., $21 \times 0 \mathrm{~mm}$., 21 de diamètre ; son centre se trouve à la distance de $1 \mathrm{~mm} ., 48$ de l'extrémité de la tête. Les testicules sont disposés dans la partie la plus postérieure du corps, ayant un diamètre de $0 \mathrm{~mm}$., 43 pour l'antérieur et $0 \mathrm{~mm}$., $49 \times 0 \mathrm{~mm}$., 45 pour le postérieur. L'ovaire se trouve juste au milieu de la longueur du corps; pour être plus précis, il faut dire que son bord antérieur se trouve juste au milieu de la longueur de l'axe du corps. L'ovaire est transversalement ovale, mesurant $0 \mathrm{~mm}$., $3 \times 0 \mathrm{~mm}$., 38 . Il est caractéristique que l'ovaire soit situé en arrière du fond de la poche du cirrhe (différence avec $T$. nematoides Mühling). La poche du cirrhe s'étend entre l'ovaire et l'orifice génital, qui se place immédiatement en avant du bord antérieur de la ventouse ventrale.

L'utérus forme des circonvolutions serrées entre l'ovaire et le testicule antérieur, n'abordant pas la partie postérieure de ce dernier ;

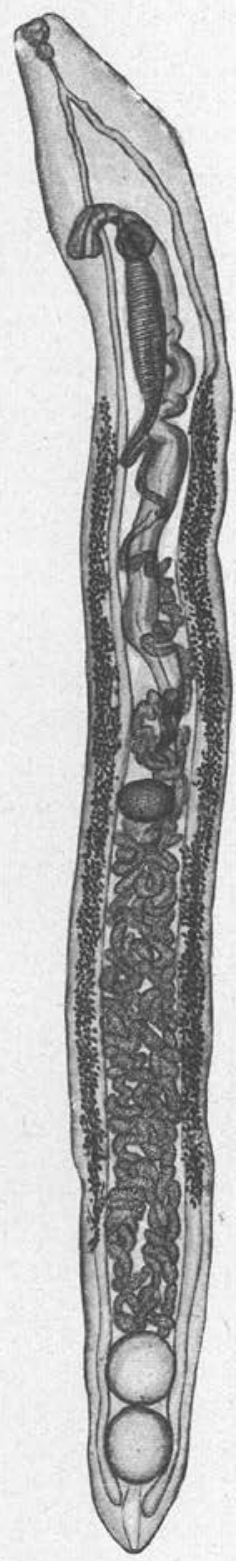

FIG. 2. - Telorchis solivagus Odhner, 1902. 
en avant de l'ovaire, l'utérus a la forme d'un canal faiblement courbé qui se dirige vers l'orifice génital. La partie terminale de l'utérus forme un métraterme puissant, avec une musculature circulaire très développée.

Une partie des glandes vitellogènes, qui sont sur deux lignes, se dispose latéralement aux branches de l'intestin, tandis que l'autre recouvre ces dernières.

Les glandes vitellogènes commencent au niveau de l'extrémité postérieure du métraterme, ce qui correspond à la limite du premier et du deuxième tiers de la distance entre la ventouse ventrale et l'ovaire.

Les glandes vitellogènes se terminent sans atteindre le testicule antérieur à une distance de $1 \mathrm{~mm}$., 92 à $2 \mathrm{~mm}$. de l'extrémité postérieure du corps. Les œufs mesurent $0 \mathrm{~mm}$., 034 de long et $0 \mathrm{~mm}$., 0193 de large. Pour la comparaison de mon exemplaire avec celui de Odhner, je donne un tableau des dimensions exprimées en millimètres.

Telorchis solivagus Odhner, 1902

\begin{tabular}{|c|c|c|}
\hline & D'APRÉs ODHNEH 1902 & D'APRÈS MES OBSERVATIONS \\
\hline Hòte ................ & Clemmys caspica Gmel. & Emys orbicularis L. \\
\hline $\begin{array}{l}\text { Distribution géographi- } \\
\text { que }\end{array}$ & Transcaucasie & \\
\hline Longueur du corps.... & 7,5 & $\begin{array}{l}\text { Transcaucasie (Armente) } \\
9,5\end{array}$ \\
\hline Largeur du corps...... & $0,8-0,9$ & 0,87 \\
\hline Ventouse buccale...... & $0,19 \times 0,24$ & $0,15 \times 0,16$ \\
\hline $\begin{array}{r}\text { Ventouse ventrale, } \\
\text { diamètre............ }\end{array}$ & 0,28 & 0,21 \\
\hline $\begin{array}{l}\text { Distance de la ventouse } \\
\text { ventrale à l'extrémité } \\
\text { antérieure du parasite }\end{array}$ & 1,5 & 1,48 \\
\hline Pharynx............ & 0,146 & 0,099 \\
\hline Diamètre des testicules & $0,5 \times 0,42$ & $0,43 \times 0,43$ et $0,49 \times 0,45$ \\
\hline Diamètre de l'ovaire... & 0,27 & $0,3 \times 0,38$ \\
\hline $\begin{array}{l}\text { Commencement des } \\
\text { glandes vitellogènes. }\end{array}$ & $\begin{array}{l}\text { A la limite entre le fer et le } \\
\text { 2e tiers de la distanee entre } \\
\text { la ventouse ventrale et } \\
\text { l'ovaire. }\end{array}$ & Mème disposition \\
\hline $\begin{array}{r}\text { Terminaison des glan- } \\
\text { des viltellogènes..... }\end{array}$ & $\begin{array}{l}\text { Au milieu, entre l'ovaire et } \\
\text { l'extrémité postérieure du } \\
\text { corps. }\end{array}$ & $\begin{array}{l}\text { Plus près des testicules } \\
\text { que de l'ovaire. }\end{array}$ \\
\hline Gufs................ & $0,031 \times 0,015$ & $0,034 \times 0,0193$ \\
\hline
\end{tabular}


Situation du parasite par rapport aUX formes voisines. - En 1885, Poirier a publié un travail contenant la description d'un trématode de l'intestin de Cistudo lutaria, qu'il prit pour Dist. gelatinosum Rud. ; Sonsino, étudiant, en 1893, la collection de trématodes du Musée de Pise, a constaté, entre le trématode décrit par Poirier et des exemplaires typiques de $D$. gelatinosum Rud., des différences qui nous forcent d'émettre quelques critiques sur l'exactitude de la description de Poirier. En outre, en 1895, Stossich, dans la révision des trématodes des reptiles, a changé le nom du parasite "D. gelatinosum Poirier (nec Rud.) " en le nommant Distomum poirieri Stoss., 1895.

En 1899, en créant le genre Telorchis, Looss a exprimé l'opinion que l'espèce décrite par Poirier était identique à Telorchis linstowi Stoss.

Braun, en 1901, étudiant les trématodes des tortues, a décrit encore une forme nouvelle d'Emys orbicularis L., à laquelle il a donné le nom de Telorchis parvus Braun.

En 1904, Stossich a donné la deseription précise accompagnée d'une figure d'un trématode trouvé par Monticelli dans l'intestin d'Emys orbicularis L. de Sardaigne (à Sassari). D'ailleurs, il explique qu'entre ce parasite et le type de Poirier, il y a quelques petites différences, qui ne l'ont pas empêché, cependant, d'identifier ces deux espèces.

En 1909, Lühe, dans sa révision des trématodes de la faune d'eau douce de l'Allemagne, décrit 2 espèces parasites d'Emys orbicularis L., qu'il avait transportés déjà du genre Telorchis dans le genre Cercochis, C. poirieri (Stoss.) et C. parvus (Braun).

A propos du premier parasite, Lühe écrit: "Im Darm von Emys orbicularis L., bisher nur einmal in Frankreich gefunden; eine ähnliche aber doch etwas abweichende Art ist im gleichen wirt bisher nur auf Sardinia beobachtet ". Cet " abweichende Art " est précisément l'espèce décrite en 1904 par Stossich.

En mettant en évidence les différences entre l'espèce de Sardaigne et celle de France, nous attirons immédiatement l'attention sur le caractère le plus important; alors que, chez T. poirieri (Stoss., 1895), les branches de l'intestin se terminent au niveau de l'espace intertesticulaire; chez $T$. poirieri, Stossich, 1904, la position de l'intestin est différente : il dépasse la limite postérieure du testicule postérieur et s'étend jusqu'à l'extrémité postérieure du corps (comme chez $T$. nematoides).

Justement, ce caractère existe chez notre espèce arménienne. Tout d'abord, j'avais même supposé que mon exemplaire arménien 
était identique à l'espèce $T$. poirieri (Stoss., 1904, nec Stoss., 1895), qui fut décrite en détail par Stossich, en 1904, et figurée.

Cependant la comparaison détaillée, nous a montré qu'à défaut d'une série d'autres caractères, l'espèce de Stossich de Sardaigne, se caractérise par les caractères suivants :

$1^{\circ}$ Sa ventouse ventrale est remarquablement plus petite que la ventouse buccale; $2^{\circ}$ l'ovaire est situé dans la moitié postérieure du corps ; alors que chez mon exemplaire : $1^{\circ}$ l'ovaire est situé juste au milieu de la longueur du corps; $2^{\circ}$ la ventouse ventrale es ${ }_{4}^{t}$ remarquablement plus grande que la ventouse buccale. Cela ne me permet pas seulement de préciser les différences entre $T$. solivagus Odhner et $T$. poirieri (Stoss., 1904, nec Stoss, 1895), mais de conclure aussi que, visiblement, $T$. poirieri (Stoss., 1895), et $T$. poirieri (Stoss., 1904), sont deux espèces différentes du genre Telorchis. Du reste, l'espèce trouvée en France par Poirier doit porter le nom de Telorchis poirieri (Stoss., 1895) et, pour l'espèce trouvée en Sardaigne, décrite par Stossich, en 1904, sous le même nom, un autre nom spécifique doit être employé.

A titre de conclusion, je donne un petit tableau synoptique des espèces de Telorchis, très voisines les unes des autres, qui habitent les tortues et les serpents d'Europe et de Transcaucasie (1).

Espèces du genre Telorchis Lühe, 1899, parasites des serpents et tortues d'Europe et Transcaucasie.

A. L'intestin atteint l'extrémité postérieure du corps.

I. L'ovaire se trouve en avant du fond de la poche du cirrhe....., T. nematoides (Mühl., 1898).

(1) Nous avons dû légèrement modifier le tableau du manuscrit de Skriabine qui était intitulé " espèces du genre Cercochis Lühe, des reptiles») et ne faisait mention que de cinq espèces, attribuées au genre Cercochis Lühe. Le genre Cercochis Lühe, 1909 (sous-genre Cercochis Lühe, 1900) a disparu de la nomenclature depuis que Stunkard (1915) a montré qu'il se confondait avec Telorchis. D'autre part, ce n'est pas cinq espèces, mais plus de 20 qui auraient dû être mentionnées par Skriabine, s'il avait voulu comprendre dans son tableau l'ensemble des espèces du genre "parasites des reptiles ». Pour ne pas trop augmenter le tableau envoyé par Skriabine, nous avons restreint le nombre des espèces à celles parasites des serpents et tortues d'Europe et de Transcaucasie, à l'exclusion des espèces (de l'ancien sous-genre Cercochis) parasites des lézards et de toutes celles d'Amérique. Dans la systématique actuelle la famille des Telorchiidx Stunkard, 1924 comprend deux sous-familles :

1. Telorchiinæ Looss, 1899 avec les genres Telorchis Lühe, 1899 [= Telorchis Looss, 1899 (nec Telorchis Lühe, 19C0)].

$2^{\circ}$ Auridistomine Stunkard, 1924, avec les genres Auridistomum Stafford, 1905, Plerygomaschalos Stunkard, 1924, Calycodes Looss, 1901, Rhytidodes Looss, 1901Dans cette dernière sous-famille Stunkard (1924) estime qu'il faut vraisemblable, ment faire entrer Cotylotretus Odhner, 1902 (= Mesaulus Braun, 1902) et Travassos propose une famille des Cotylotretide Trav., 1922. Mais, pour Odhner (1910, p. 96, 97, 163), Cotylotretus, malgré l'absence de crochets céphaliques, appartient en réalité à la grande famille des Echinostomida, avec laquelle Rhytidodes a des affinités indiscutables mises en évidence par Braun (1901, p. 31-33) et Odhner (1910, p. $163-164$.

[Robert-Ph. Dollfus]. 
II. L'ovaire se trouve au niveau de la poche du cirrhe....., T. aculeatus (von Linstow, 1879) $=T$. linstowi (Stossich, 1890).

III. L'ovaire se trouve en arrière du fond de la poche du cirrhe.

a) La ventouse buccale est environ deux fois plus grande que la ventouse ventrale....., T. poirieri (Stoss., 1904, nec Stoss., 1895).

b) La ventouse buccale est à peine plus grande que la ventouse ventrale ou subégale....., T. ercolanii (Monticelli, 1893).

c) La ventouse ventrale est plus grande que la ventouse buccale....., T. solivagus, Odhner, 1902.

B. L'intestin ne dépasse pas la limite du testicule postérieur.

I. Les glandes vitellogènes commencent avant l'ovaire....., T. poirieri (Stossich, 1895, nec Stoss., 1904) (1).

II. Les glandes vitellogènes commencent après l'ovaire....., T. parvus (Braun, 1901). Moscou, 10, IV, 1924.

(1) C'est-à-dire Telorchis stossichi Goldberger, 1911. Un nom nouveau que nous avons dû supprimer comme tombant en synonymie, se trouvait dans le manuscrit de Skriabine, cet auteur n'ayant pas eu connaissance du nom proposé par Goldberger.

[Robert-Ph. Dollfus]. 